\title{
An Average Power Reduction Method for Web Applications on Wireless Terminals
}

\author{
Toshiya Koyasu, Hideki Shimada, Kenya Sato \\ Department of Information Systems Design, Doshisha University, Kyoto, Japan \\ Email: t.koyasu35880@gmail.com, hideki-s@is.naist.jp, ksato@mail.doshisha.ac.jp \\ Received February 11, 2012; revised March 20, 2012; accepted April 17, 2012
}

\begin{abstract}
Recently, there is a widespread use of Web browser-based Web applications such as e-mails and chats. However, frequent communication between mobile wireless terminals and HTTP servers give rise to problematic increases of average power consumption for the mobile devices. Current attempts to tackle this problem focus on reducing power consumption at the network and data link layers used by the devices. In this study, we propose a different solution where certain functionalities of the mobile application are delegated to other devices with abundant power resources (either from large capacity batteries or power outlets). This method off-loads parts of the communication process normally done at wireless terminals to amply powered machines. With messages pushed from the machines only when the HTTP server responds with an update, the wireless terminal needs to transmit data less frequently, thus cutting down on power consumption. Our prototype implementing the proposed method succeeded in reducing the rise in average power consumption.
\end{abstract}

Keywords: Web Applications; Ajax; Wireless Communication

\section{Introduction}

Recent years have seen a growth of smart phones and other wireless devices with sophisticated Web browsers [1], making it possible and more practical to use Web pages and applications from wireless terminals. This has also made common the use of Ajax applications from wireless terminals. Ajax applications are notable for their high usability and their convenience of not having to install additional software. Some examples of these are text-based chats and email applications that let users communicate using the Web browser as the interface.

However, the use of Web applications with Ajax technology on a wireless terminal, ones that synchronize data updates on the server with Web browser displays, poses a problem: High power consumption. In this study, we propose a method of reducing power consumption at the wireless terminal while using Ajax [2] applications.

\section{Device Power Consumption}

\subsection{Web Applications}

Ajax is an approach to Web applications that attempts to improve on usability. Web application usability is tied to the way the browser and the HTTP server communicate with each other: a critical functionality for a Web application. Traditional Web applications without Ajax would reload the whole displayed page every time this needs to be done. This action causes unfavorable user experience because it temporarily removes UI components such as text boxes and buttons from the display, and could possibly interrupt the user's tasks. On the other hand, Ajax applications only update parts of the display, only when they need to be updated. UI components remain visible, which leads to higher usability.

Figure 1(a) shows an example of how an Ajax application works. In this application, the user types a product ID in the ID text box and presses the SEARCH button. A search is then conducted in the remote database for the corresponding product. The result is displayed in the NAME text box while other parts of the UI remain as they are. Figure 1(b) is the sequence of actions beginning with the ID input and ending with the product name display.

- Web browser: The front-end of the Web application.

- HTTP Server: A collection of Web, application and database servers that is the back-end of the Web application.

- XHTML File: The file that defines the visuals and functionalities of the Web application.

- Javascript: The scripting language used to embed extended functionalities on the Web page.

- Ajax engine: The Javascript program (a group of functions) that provides HTTP communication and UI 
(a) Appearance of the Ajax Application

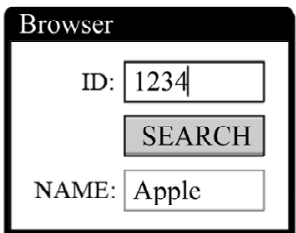

(b) Sequenec of the Ajax Application

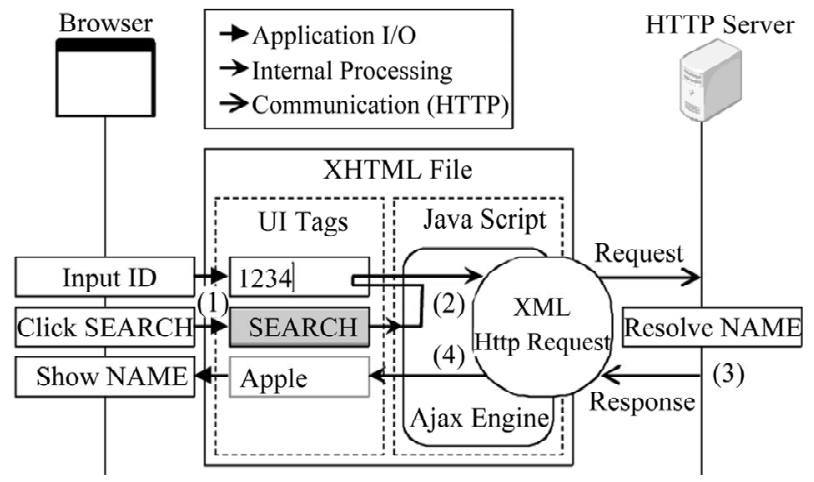

Figure 1. Behavior of the Ajax application.

updating.

- XML Http Request: An HTTP client library that can be used from a Javascript program.

- UI Tags: The XHTML tags that define the Web application's UI.

The basic components and actions 1) through 4) in the figure are as follows:

1) The user types the product number in the ID text box and presses the SEARCH button. A function defined in the Ajax engine is called as an event handler for the button press event.

2) The function retrieves the ID text box value and sends it to the server using XML Http Request. At the same time, another Ajax engine function is registered as a handler for the event that occurs when a response is received.

3) The HTTP Server resolves the product name based on the value received, and sends back a response to XML Http Request.

4) XML Http Request calls the event handler registered in action 3). The handler inserts the product name into the NAME text box.

\subsection{Synchronous Applications}

An application where browser display updates are synchronized with server data updates, such as a browserbased chat application, would update the browser display to show a newly submitted message when it is posted to the HTTP server. We call this type of application a "synchronous application”. The Web being a pull-based communication system, a synchronous application would need to somehow send request messages periodically from the browser to the HTTP server. We will take the chat application as an example to describe how these messages can be sent.

1) Non-Ajax method

B-Chat [3] achieves periodical requests with client pull, a feature in which XHTML files are re-retrieved and redisplayed periodically. B-Chat uses this feature to get the XHTML file with the latest messages every once in a while. With client pull, the whole browser display must be reloaded every time synchronization occurs, so the user wouldn't be able to operate on the page if the sync interval is too short. Therefore, applications that use client pull tend to set the sync interval to longer periods compared applications using Ajax. B-Chat uses a 30 second sync interval.

2) Ajax method

Ajax Chat [4] achieves periodical requests using Javascript functions in its Ajax engine. Applications using this method can set shorter sync intervals because only specific parts of the browser display needs to be updated. The user is not interrupted by synchronization. Rather, the shorter the sync interval, the smaller the latency of display update after data is updated on the server, so applications that use Ajax tend to set shorter sync intervals compared non-Ajax applications. Ajax Chat uses a 2 second sync interval.

Mobile wireless terminals consume more power when communicating via its wireless network interface. A study [5] done by Carroll et al. shows that a particular device's power consumption while communicating was $1016.4 \mathrm{~mW}$, which was 6.31 times higher than while off communication (161.2 mW). We can generally say that using an application with a high rate of communication on a wireless terminal increases its energy consumption. A synchronous application is one of them. Ajax applications in particular communicate more frequently compared to others, so the problem is grave and needs to be addressed.

\section{Proposed Method}

\section{Approach}

To reduce average power consumption on a device using synchronous Ajax applications, we propose the two following approaches to off-load transactions and reduce communication frequency.

- Delegating communication to other machines:

We use other devices to carry out the actual communications for the wireless terminal while data updates are less frequent. We call this other device the "Surrogate Device”. Devices fit for this role are ones with network connections that have large capacity batteries or enjoy access to steady power from outlets. Some 
examples would be desktop PCs with their screen savers running, idle servers, and networked home appliances [6] that are to be introduced in the near future.

- Using push-based communications to deliver updates: We use push-based communications to send updated data to the wireless terminal after the Surrogate Device retrieves them from the HTTP server. In pushbased communications, the data is sent from the publisher to the recipient without the recipient requesting it. Thus the wireless terminal does not need to poll the Surrogate Device for updates.

Figure 2 shows the proposed communication model for this transaction off-loading. Descriptions of the communications 1) through 4) are as follows:

1) Direct communication: This phase is used to detect communication done by synchronous Ajax applications against the HTTP server. After a communication is detected, the system checks to see if there are updates at the HTTP server. If the data is updated frequently at the remote server, the wireless terminal continues to communicate directly with the server, as off-loading would only have trivial effects. This also avoids the overhead time produced by off-loading. The same is done for non-Ajax synchronous applications; they communicate directly with the HTTP server.

2) Surrogate Communication Request: If data updates at the HTTP server are infrequent, then the wireless terminal requests the Surrogate Device to carry out the actual communications. We call this request the "Surrogate Communication Request", and the message sent at this time the "Surrogate Communication Request Message". The wireless terminal suspends communications after sending out this request.

3) Surrogate communication: The Surrogate Device, upon receiving a Surrogate Communication Request, checks the HTTP server for data updates.

4) Surrogate Communication Response: If data is updated on the remote server, the Surrogate Device ends

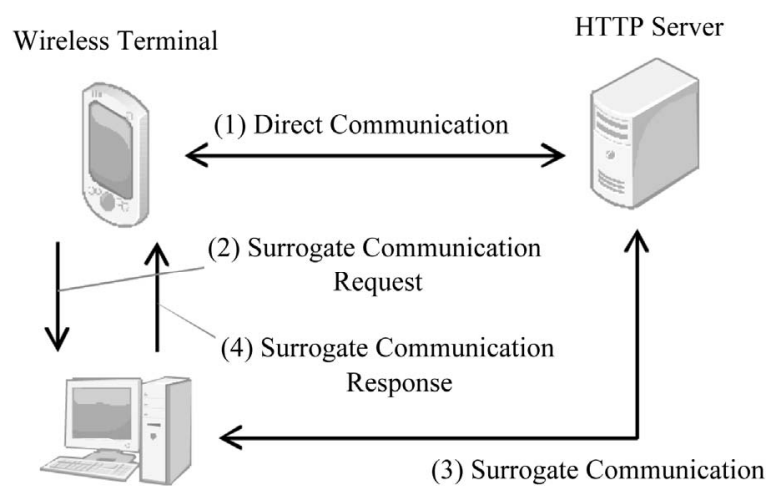

Surrogate Device

Figure 2. Communication model of the transaction offload. communications with the HTTP server and pushes the updated data back to the wireless terminal. We call this push the "Surrogate Communication Response" and the message sent at this time the "Surrogate Communication Response Message”.

\section{Prototype Implementation}

Figure 3 shows the setup of the prototype we implemented to evaluate the proposed method. Each of the components are shown corresponding to Figure 2. The prototype consists of the Master Gateway (MGW), residing on the wireless terminal, and the Slave Gateway (SGW), residing on the Surrogate Device.

\subsection{Master Gateway}

The MGW is the module to be used by the browser as a local proxy server. As with a generic local proxy server, the MGW relays communications between the browser and the HTTP server and caches the server responses. In addition to this basic functionality, it also distinguishes the transactions that are taking place. This is done based on the request URI after stripping it of the query parameters. Each of the transactions is assigned a defined state. The state assigned to a transaction is checked when the browser sends a request. Subsequent behavior of the MGW depends on the state of each of the transactions. The four states and their corresponding actions are as follows:

- Ajax Communication Detection State AJAX_DET: In the initial state, AJAX_DET, the MGW scans the communications between the browser and the HTTP server and detects those that are done by Ajax applications. This is done by finding JSON objects (packages of data often used with Ajax applications) in the HTTP server's response messages. The MGW finds JSON objects by checking the Content-Type header in HTTP responses. Once the MGW detects an Ajax communication, the state transitions to UPSTOP DET.

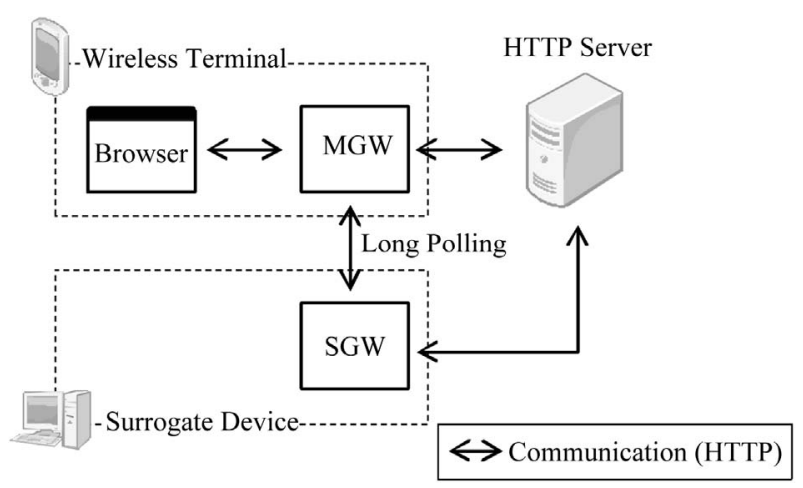

Figure 3. Components of the prototype. 
- Update Stop Detection State (UPSTOP_DET): In the UPSTOP_DET, the MGW attempts to detect whether updates at the HTTP server has ceased temporarily. The MGW decides that updates have ceased temporarily when the latest response from the HTTP server is identical to the most recently cached response. Once a temporary cease in communications has been detected, the MGW sends a request message to the SGW (the Surrogate Communication Request Message) that contains both the browser's request and the server's response, combined as a multi-part message body. This is the Surrogate Communication Request Message. The state now transitions to RES_WAIT.

- Response Waiting State (RES_WAIT):

In RES_WAIT, the MGW waits for a response from the SGW. Any Ajax requests sent from the browser are responded with cached contents. Completing all communications within the wireless terminal in this way has the effect of practically suspending its communications. Once the SGW responds with a Surrogate Communication Response Message, the cache is overwritten with the newly arrived data. The state now transitions to RESD.

- Surrogate Response Delivery State (RESD):

In RESD, the MGW delivers the Surrogate Communication Response Message to the browser. The message, which is the cache contents that have been overwritten, is delivered in response to requests from the browser. The state transitions back to UPSTOP_DET at this point.

\subsection{Slave Gateway}

The SGW is the module that receives Surrogate Communication Requests from the MGW and carries out the communications for the wireless terminal; it sends out the request message contained in the Surrogate Communication Request Message periodically to the HTTP server. Consecutive responses from the server are checked to see if they differ from the sample response message contained in the Surrogate Communication Request Message. If in fact the responses do differ, the SGW decides that the remote data has been updated, and pushes the new response (the Surrogate Communication Request Message) to the MGW and terminates communications with the server.

\subsection{Method of Content Transmission}

For content transmission from the SGW to the MGW, we adopt the long polling method, which is used to achieve pseudo-pushing in pull-based communication systems such as the Web. In long polling, the client initially sends a request to the server. The server, instead of responding instantly, holds the request until a certain event occurs (usually the arrival of newly available data). Once this event occurs, the server sends a response to the client.

In this prototype, the SGW holds the Surrogate Communication Request until it detects data updates at the server.

\section{Evaluations}

\subsection{Evaluation Items}

To evaluate the proposed method, we implemented and ran a sample application (described later) in various situations for 10 minutes each and evaluated the following two points.

- The Number of transmissions by the wireless terminal: We compared the number of times the wireless interface was used to send and receive messages.

- Average latency: We compared the average latency of the browser display update since the data update on the server.

\subsection{Evaluation Method}

We used an application resembling the communication model of an Ajax synchronous application, the chat. This application has the following two front-ends.

1) Sender

The Sender front-end is the one that submits new chat messages. It submits messages at normally distributed random time intervals having an average of $\mu$ (msec.) and a standard deviation of 250 (msec.). The requests have two parts to its query parameter. One is a timestamp of the request (the number of milliseconds since January 1st, 1970 at 00:00) that represents the time of submission of the chat message. The other is a sequence of 140 randomly selected Japanese Hiragana characters, encoded in UTF-8, that represents the actual chat message.

2) Receiver

The Receiver front-end is the one that retrieves submitted chat messages. It sends out a request to the HTTP server once every 3 seconds. The request has the timestamp of the request as the query (in the same format as the Sender). The HTTP server responds with chat messages that were submitted later than this timestamp.

\subsection{Method of Content Transmission}

Table 1 shows the combinations of evaluation conditions,

Table 1. Evaluation cases.

\begin{tabular}{ccccc}
\hline & case 1 & case 2 & case 3 & case 4 \\
\hline$\mu$ (msec) & 3000 & 3000 & 9000 & 9000 \\
$T$ (msec) & n/a & 1500 & n/a & 1500 \\
\hline & case 5 & case 6 & case 7 & case 8 \\
\hline$\mu$ (msec) & 9000 & 27000 & 27000 & 27000 \\
$T$ (msec) & 3000 & n/a & 1500 & 3000 \\
\hline
\end{tabular}


consisting of $\mu$ (the average time intervals between message submissions by the Sender) and $T$ (the time intervals between surrogate communications by the surrogate communication device). $T$ is shown as "n/a" where this method is unnecessary.

\subsection{Method of Content Transmission}

Specifications of each device used in the evaluation are shown in Tables 2-4. The devices all reside in different networks, and the Sender and Receiver are used on the wireless terminal.

During evaluation, we turned off the wireless device's HSDPA interface and used its IEEE $802.11 \mathrm{~g}$ interface, and limited its throughput to that of HSDPA, which we had measured in advance. This is in consideration of the possibility that the HSDPA interface's unstable throughput may cause unpredictable effects on evaluation. We used Iperf 1.7.0 [7] with a window size of $32 \mathrm{~KB}$ to measure the HSDPA interface's through put. The limit applied to the IEEE $802.11 \mathrm{~g}$ interface is the average of 20 transmissions between the wireless terminal and the HTTP server. The actual values are $80 \mathrm{Kbps}$ upstream and $40 \mathrm{Kbps}$ downstream. We used NEGiES 1.57 [8] to apply the throughput limits.

\subsection{Evaluation Procedure}

First, the Receiver starts retrieving chat messages. Once the first response from the HTTP server is retrieved, the Sender starts submitting chat messages. Both front-ends are stopped after 10 minutes (counting from the initial message retrieval by the Receiver). The number of transmissions by the wireless terminal and the average latency are then evaluated.

Table 2. Specification of the wireless terminal.

\begin{tabular}{cc}
\hline CPU & Atom Z520 1.33 GHz \\
\hline Memory & $1.00 \mathrm{~GB}$ \\
Network & HSDPA [9], IEEE 802.11 g \\
Web Browser & Firefox 3.6.13 \\
\hline
\end{tabular}

Table 3. Specification of the surrogate device.

\begin{tabular}{cc}
\hline CPU & Core2 Duo U9400 $1.40 \mathrm{GHz}$ \\
\hline Memory & $3.00 \mathrm{~GB}$ \\
Network & 100 BASE-TX \\
\hline
\end{tabular}

Table 4. Specification of the HTTP server.

\begin{tabular}{cc}
\hline CPU & Core 2 Duo E7500 $2.93 \mathrm{GHz}$ \\
\hline Memory & $2.00 \mathrm{~GB}$ \\
Network & 100 BASE-TX \\
\hline
\end{tabular}

\subsection{Evaluation Results}

The resulting number of communications and the average latency are shown as follows: cases 1 and 2 in Figures 4 and 5, cases 3 through 5 in Figures 6 and 7, cases 6 through 8 in Figures 8 and 9.

\section{Discussion}

Let us define the average increase in the wireless terminal's power consumption, compared to when the terminal's communications are halted, as

$$
w_{\text {avg }}=\left(W_{\text {com }}-1\right) \frac{t_{c o m}}{t} .
$$

Here, $W_{\text {com }}$ is the rate in which power consumption increases or decreases when communicating, compared to when it is not. We set this according to the study by Carroll et al. (cited in Chapter 2) to

$$
W_{\text {com }}=6.31
$$

it is the length of time the sample application ran, which is

$$
t_{\text {com }}=10 \times 60=600
$$

$t_{\text {com }}$ is the total length of time (sec) the Receiver used the wireless terminal's interface to communicate.

Figure 10 shows the values of Equation (1) when applied to the evaluation results for cases 3 through 5. Figure 11 is for cases 6 through 8 .

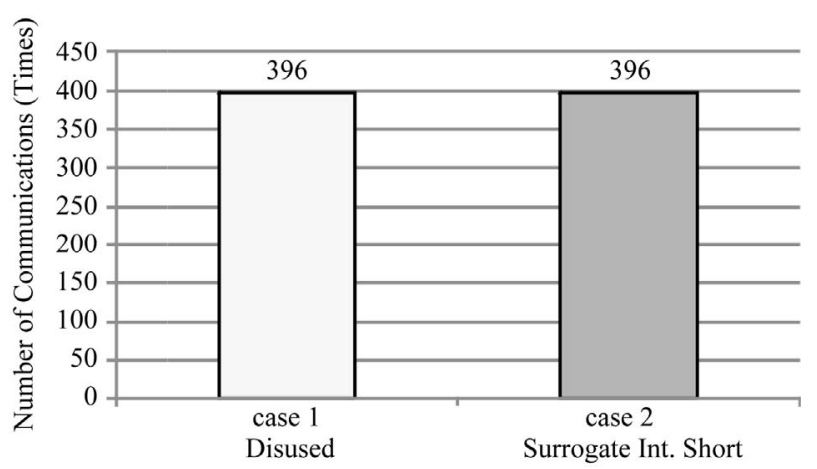

Figure 4. Number of communications on cases 1 and 2.

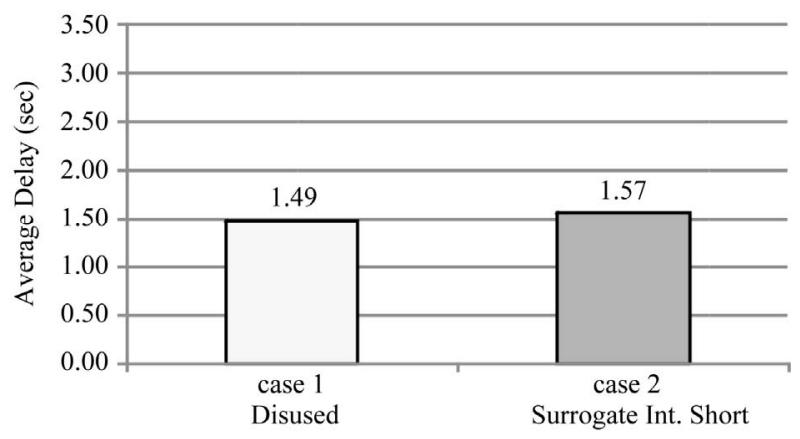

Figure 5. Average delay on cases 1 and 2. 


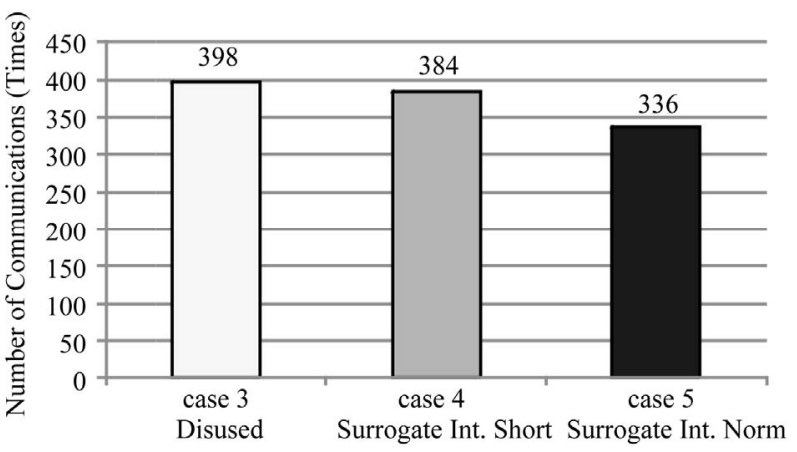

Figure 6. Number of communications on cases 3 to 5 .

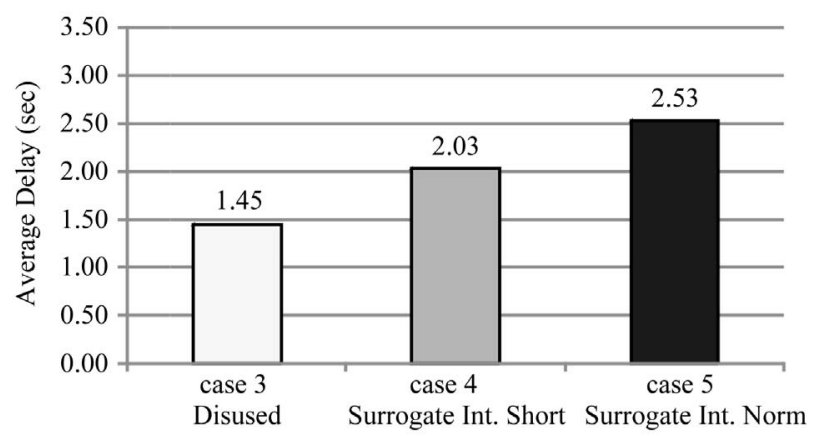

Figure 7. Average delay on case 3 to 5.

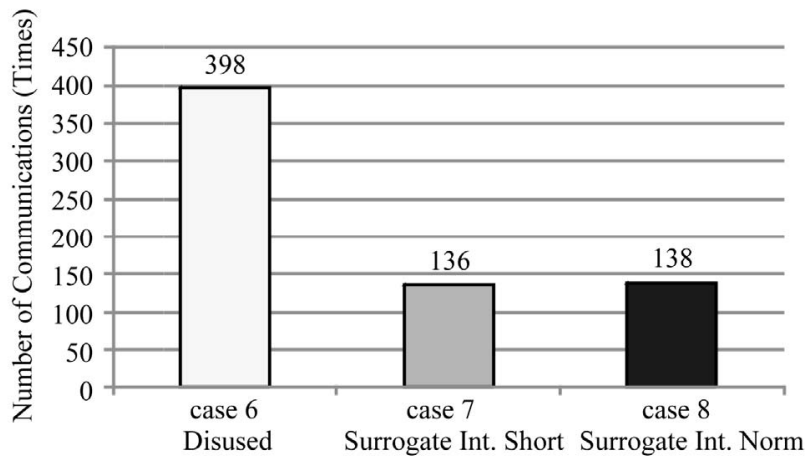

Figure 8. Number of communications on cases 6 to 8 .

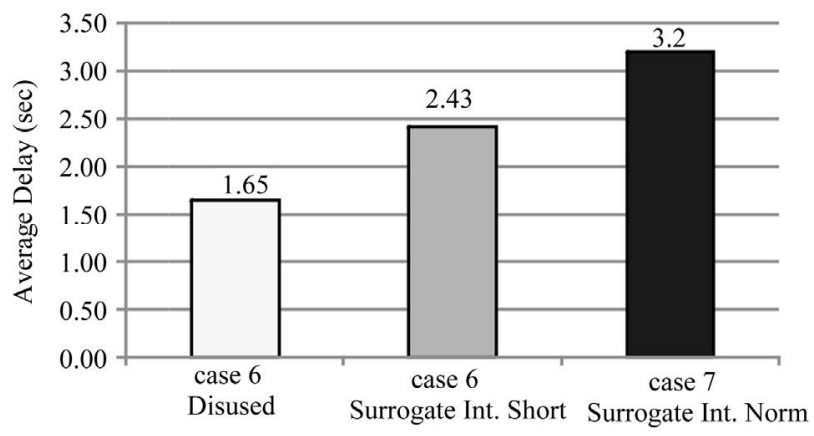

Figure 9. Average delay on cases 6 to 8 .

From Figure 11, we can see that the wireless terminal consumed $14 \%$ more power when using the sample

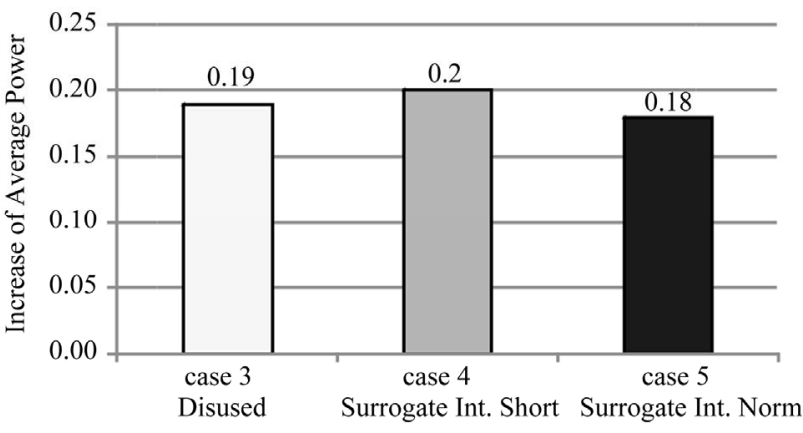

Figure 10. Increase of average power on cases 3 to 5 .

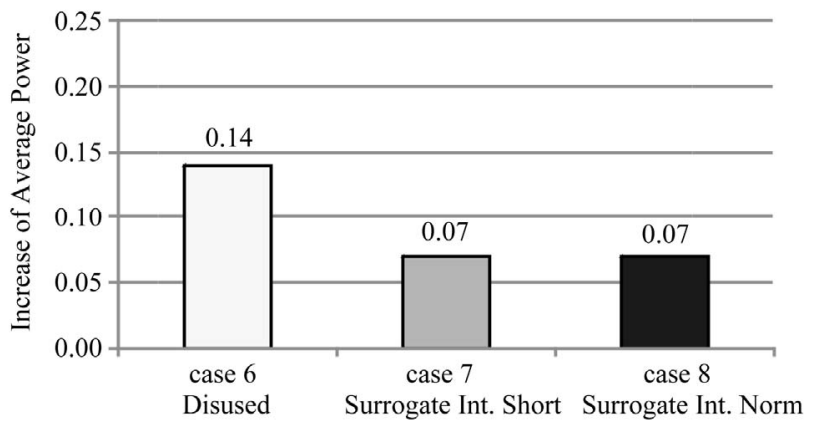

Figure 11. Increase of average power on cases 6 to 8.

application (case 6) and that our proposed method lowered it to 7\% (cases 7 and 8). However, in Figure 10, the average power consumption when not using our method is $19 \%$ (case 3 ), which is lower than the $20 \%$ when it was used (case 4).

There are two possible reasons for this. One is that the HTTP server's data update rate (approximately once every 9 seconds) was relatively low for the sync rate (approximately once every 3 seconds). As such, the number of transmissions eliminated was a trivial 14 times. The other is that the size of the Surrogate Communication Request Message (963 bytes) was larger than that of the data update request message in long polling (434 bytes) and the wireless terminal had to communicate for a longer total time.

In summary, the proposed method can reduce power consumption given that data update intervals are sufficiently long compared to the sync interval of the synchronous application. More specifically, we can conclude that our method is effective if the data update intervals are more than 9 times longer than the sync interval.

\subsection{Overhead Time}

Figure 5 shows that when surrogate communications do not occur (case 2), our method has an increased average overhead time of under $100 \mathrm{~ms}$ compared to when our method is not used (case 1), which is acceptable. Figures 7 and 9 show that when surrogate communications do occur (cases 4, 5, 7 and 8), the increased average over- 
head time can be held down to under 1 second if the intervals of surrogate communications are approximately half of the sync interval.

With the above evaluation results, combined with the fact that synchronous applications do not impose realtime constraints, we conclude that the overhead time introduced by our proposed method does not impair practicality.

\subsection{Comparisons with Related Methods}

In the method proposed by Ishida et al. [10], the wireless interface on the terminal is set to sleep mode every time a transmission ends. A device called the Wakeup Module is then used in place of the wireless interface to wait for incoming transmission waves and wake up the wireless interface when needed.

In the method proposed by Russel [11], the front-end and the back-end of the Ajax application are modified to adapt long polling (described in Section 4.3) to reduce the communication rate: The HTTP server waits until there is new data available to respond to the browser.

Both of these methods require specific expertise in mobile devices or Web applications if they are to be applied to existing applications. As such, it is difficult if not impossible for the end user to use those methods. In contrast, our method has the advantage of being able to be used by the end user, since it does not require that modifications be made on existing applications.

\section{Conclusions}

In this study, we proposed a method of off-loading a wireless terminal's communications to another device in order to tackle the problem of increased power consumption that occurs while using synchronous Ajax applications (applications that synchronize the Web browser's display with data updates on the server). Our evaluation prototype kept the average increase in power consumption.

We used a networked device as the Surrogate Device in this particular study, but this method could potentially be adapted to wireless base stations and Web servers themselves.

Our method is a valuable contribution in the application software level to the wide social needs [12] of longer battery lives. Because this is a high-level method, we can expect it to be used in conjunction with other low-level technologies in hardware and communications.

\section{Acknowledgements}

This work was supported by JSPS Grant-in-Aid for Scientific Research KAKENHI (2100084).

\section{REFERENCES}

[1] M. Stanley, “The Mobile Internet Report,” 2009. http://www.morganstanley.com/institutional/techresearch/ mobile_internet_report122009.html

[2] J. J. Garrett, “Ajax: A New Approach to Web Applications,” 2005.

http://www.adaptivepath.com/ideas/ajax-new-approach-w eb-applications

[3] http://www.adaptivepath.com/ideas/essays/archives/0003 85.php

[4] http://wws.cside.com/cgi-plant/b_chat/blueimp.net, https://blueimp.net/ajax/

[5] A. Carroll and G. Heiser, "An Analysis of Power Consumption in a Smart Phone," Proceedings of the 2010 USENIX Annual Technical Conference, Boston, 23-25 June 2010, pp. 1-14.

[6] M. Isshiki, M. Hirahara and T. Kishimoto, "FEMINITY Series Home Network System for Network Home Appliances,” Toshiba Review, Vol. 57, No. 10, 2002, pp. 7-10.

[7] NLANR/DAST, “Iperf 2.0.3,” 2008. http://iperf.sourceforge.net/

[8] RHOX, “NEGiES Version 1.57," 2005. http://hp.vector.co.jp/authors/VA036210/

[9] Japan Communications Inc., “B-Mobile3G,” 2009. http://www.bmobile.ne.jp/english/3g.html

[10] S. Ishida, M. Suzuki, T. Morito and H. Morikawa, “A Multi-Step Wake-Up Scheme for Low-Power-Listening Wireless Communication System," Technical Report of IEICE, Information Network, Vol. 107, No. 525, 2008, pp. 355-360.

[11] A. Russel, “Comet: Low Latency Data for the Browser," 2006.

http://infrequently.org/2006/03/comet-low-latency-data-f or-the-browser/

[12] ORIMO Inc., "ORIMO Mobile Research, a Study on Smart Phones,” 2010. http://www.orimo-r.co.jp/upload_2/gif/phone0601.pdf 